\title{
BER Performance of OFDM-BPSK and -QPSK Over Generalized Gamma Fading Channel
}

\author{
Neetu Sood \\ Department of Electronics and \\ Communication Engineering \\ National Institute of Technology \\ Jalandhar,India
}

\author{
Ajay K Sharma \\ Department of Computer Science \\ Engineering \\ National institute of Technology \\ Jalandhar, India
}

\author{
Moin Uddin \\ National Institute of Technology \\ Jalandhar \\ India
}

\begin{abstract}
In this paper, the BER performance of OFDM-BPSK and -QPSK system over generalized gamma fading channel has been reported. This model is versatile enough to represent short-term fading such as Weibull, Nakagami-m, or Rayleigh as well as shadowing. The flexibility of this model is because of two fading parameters compared to only one in Nakagami-m fading model, which helps to analyze the severity of fading more deeply. Here, simulations of OFDM signals are carried with generalized gamma faded signal to understand the effect of channel fading.
\end{abstract}

\section{Keywords}

OFDM, Fading distribution, Nakagami-m channel, Generalized Gamma fading.

\section{Introduction}

OFDM technique is a multi-carrier transmission technique, which is being recognized as an excellent method for high-speed bidirectional wireless data communication. The prime idea is that all queuing data in buffer are uniformly allocated on small sub-carriers. OFDM efficiently squeezes multiple modulated carriers tightly together reducing the required bandwidth but keeping the modulated signals orthogonal so that they do not interfere with each other. OFDM that is highly efficient technique shows favorable properties such as robustness to channel fading and inter symbol interference (ISI) and is more immune to noise. OFDM system is capable of mitigating a frequency selective fading channel to a set of parallel flat fading channels, which need relatively simple processes for channel equalization.

Rayleigh and Rician fading distributions in frequency selective fading channels have already been deployed and studied in depth for OFDM systems [1,2]. Various channel estimation and diversity schemes have been proposed in literature to enhance the error performance under Rayleigh and Rician fading channel [3-5]. Nakagami-m fading distribution is another useful and important model to characterize the fading channel [6]. Kang et al. [7] modeled the OFDM- BPSK system with frequency selective fading channel. Zheng et al. [8] further enhanced the work by presenting asymptotic BER performance of OFDM system in frequency selective Nakagami-m channel. In [9], accurate error performance of OFDM systems is analyzed on basis of number of channel taps in Nakagami-m fading environment.

OFDM systems have gained an equivalent attention with flat fading environment. In [10], present the method of channel estimation and carrier frequency offset to design an OFDM receiver in flat fading environment. However, BER performance of OFDM system in flat fading channel using DBPSK modulation technique is studied by Lijun et al. [11].The influence of gaussian and flat Rayleigh fading channel on the PAPR of MIMO-OFDM passband signals at the receiver-side have been presented in [12].

Another versatile wireless channel model, which can generalize the commonly used models for multipath fading and shadowing, is the two-parameter generalized gamma model [13]. It includes multipath fading models such as Rayleigh, Nakagami-m, and Weibull as special cases and lognormal shadowing model as the limiting case. The performance of DS-CDMA in the frequency selective time non-selective multipath Generalized Gamma fading channels has already been been investigated in [14]. However, performance measures such as the amount of fading, average bit error rate, and signal outage for generalized gamma fading channel has been presented in [15]. So, our motivation behind this paper was to study the performance analysis of generalized gamma fading for OFDM system.

This rest of the paper is organized as follows: In the following section, OFDM system model is described. In section 3 , the mathematical model to generate OFDM received signal and the envelope of generalized gamma fading channel is discussed. Section 4 conainns the analysis of simulated results of performance of OFDM system is under the influence of Gamma fading. Finally section 5 , concludes the paper.

\section{Model Description}

A Complex base band OFDM signal with $\mathrm{N}$ subcarriers is expressed as [16]:

$$
s(t)=\sum_{k=0}^{N-1} D_{i} e^{j 2 \pi k f_{0} t} \quad 0 \leq t \leq T
$$

For each OFDM symbol, the modulated data sequences are denoted by $D(0), D(1), \ldots . D(N-1)$. Here, $f_{0}$ denote the sub-carriers spacing and is set to $f_{0}=1 / T$ the condition of orthogonality. After IFFT, the time-domain OFDM signal can be expressed as [16]:

$$
\begin{aligned}
s(t)= & \frac{1}{N} \sum_{k=0}^{N-1} D_{i} e^{\frac{j 2 \pi k f_{0 n}}{N}} \\
& =\operatorname{IFFT}\left(D_{0}, D_{1}, \ldots, D_{N-1}\right)
\end{aligned}
$$


After IFFT, the modulated signal is up-converted to carrier frequency $f_{C}$ and then the following signal is produced and transmitted through channel [16]:

$x(t)=\operatorname{Re}\left\{\sum_{k=0}^{N-1} D_{i} e^{j 2 \pi k\left(f_{0}+f_{C}\right) t}\right\} \quad 0 \leq t \leq T$

$x(t)$ represents the final OFDM signal in which sub-carriers shall undergo a flat fading channel.

\section{Channel Model}

In this paper, the sub-channel spacing $f_{o}=1 / T$ is chosen so that the produced parallel fading sub-channels have flat fading characteristics. So, we have chosen Generalized Gamma flat fading channel with additive white Gaussian noise. In flat fading environment, the base-band signal at the input of receiver $y(t)$ is as described as follows:

$$
y(t)=x(t) * r(t)+n(t)
$$

where, $x(t)$ denotes the base-band transmitted signal, $r(t)$ is the Generalized Gamma distributed channel envelope and $n(t)$ is the additive white Gaussian noise with zero mean.

Generalized Gamma fading distribution function is given as:

$r(t)=\frac{2 v r^{2 v m-1}}{\Gamma(\mathrm{m})(\Omega / \mathrm{m})} \exp \left(-\frac{m r^{2 v}}{\Omega}\right) \quad r \geq 0$

where $\mathrm{v}>0$ and $\mathrm{m}>0$ are fading parameters, $\Omega$ is the scaling parameter and $\Gamma($.$) is the Gamma function. Superior fit to the$ measured data over a wide range of physical channel conditions is presented in [17].

Nakagami- $\mathrm{m}$ fading distribution function is given by [6]:

$$
p_{R} \quad r=\frac{2 m^{m} r^{2 m-1}}{\Gamma(m) \Omega^{m}} \exp \left(-\frac{m r^{2}}{\Omega}\right), r \geq 0
$$

where, $\Gamma($.$) is the Gamma function, \Omega=\overline{r^{2}}$ is the average power, $m$ is fading parameter and $r$ is Nakagami distribution envelope.

The fact that generalized gamma distribution has one more parameter than the well known distributions renders it more flexible to better adjust with measurement data. Moreover, this model is based on more realistic heterogeneous scattering environment. For wireless systems, generalized gamma model provides a simple way to model all forms of channel fading conditions including shadowing. By varying the two parameters $\mathrm{v}$ and $\mathrm{m}$, different fading and shadowing conditions can be described.

For instance, $v=1$, in Eq. 5 represent Nakagami-m fading;

$\mathrm{m}=1$, Eq. 5 represent Weibull fading;

$\mathrm{m}=\mathrm{v}=1$, Eq. 5 represent Rayleigh fading.

In performance analysis of modulations techniques over flat-fading channels only the knowledge of the envelope statistics is required and the phase is assumed to be uniformly distributed. Based on the theoretical description given, the Nakagami-m envelope variates can be generated in a simple and efficient way using the following equation:

$r(t)=\left(\sqrt{\sum_{\mathrm{n}=1}^{\mathrm{m}}\left(\mathrm{p}^{\mathrm{n}}\right)^{2}+\sum_{\mathrm{n}=1}^{\mathrm{m}}\left(\mathrm{p}^{\mathrm{n}}\right)^{2}}\right)$

Where, $\mathrm{p}^{(\mathrm{n})}$ and $\mathrm{q}^{(\mathrm{n})}$ are independent Gaussian distributed with zero mean and identical variance.The received envelope at any point is assumed to consist of m number of multipath components and the non-linearity of this heterogeneous environment represented in the form of an exponent $1 / \mathrm{v}$, so that the resultant generalized gamma distributed envelope of can be generated using the following equation:

$r(t)=\left(\sqrt{\sum_{\mathrm{i}=1}^{\mathrm{m}} \mathrm{p}_{\mathrm{i}}^{2}+\sum_{\mathrm{i}=1}^{\mathrm{m}} \mathrm{q}_{\mathrm{i}}^{2}}\right)^{1 / \mathrm{v}}$

Where , $\mathrm{p}_{\mathrm{i}}$ and $\mathrm{q}_{\mathrm{i}}$ are independently distributed Gaussian variables with zero mean and unit variance. Equation 8 is valid for only discrete value of $m$ [13]. Equation (7) and (8) clearly indicates the relationship between nakagami-m envelope and generalized gamma distributed envelope, which can also be obtain by using PDF equations of both the fading distributions.

Nakagami-m and Generalized Gamma fading distributions shares a relationship of non-linearity that depends upon the factor $1 / v$.

So, it is clear that performance of OFDM system under the influence of Nakagami-m fade will lead to analysis of the OFDM system under Generalized Gamma fade.

Since, it is mentioned in the literature that there exist a threshold value of $m$, so firstly, the simulations are conducted to determine this threshold value of $m$. Then, simulations are carried for the generalized gamma fading channel by keeping the value of $m$ to be optimal and varying the value of $v$.

\section{Results and disscussions}

\section{Case I: Under the influence of Nakagami-m fade}

In this section, we have presented the simulation results using MATLAB $^{\mathrm{TM}}$ by implementing two modulation formats for OFDM to get threshold value of fading parameter $\mathrm{m}$. Figure 2 indicates the BER versus SNR for OFDM-BPSK with different values of fading parameter $\mathrm{m}$. It is clear from section 3 , that at $\mathrm{m}=1$, Nakagami-m fading corresponds to Rayleigh fading. So, the results for the same have been achieved through simulations. Nakagami-m PDF with parameter $m<1$ models worse than Rayleigh conditions and fading becomes more severe. So, the value of $m$ is increased and as a result the BER starts reducing and value of $10^{-4}$ is reported at $\mathrm{m}=$ 1.4, 1.5 and 2. Further, if we increase $\mathrm{m}$, no reduction in BER has been reported rather it starts increasing. So this put a limit to increase the value of $m$ beyond the certain value. The optimal value of $\mathrm{m}$ is achieved to be 1.4, to estimate the fading channel. However, it is commonly believed that larger the value of $\mathrm{m}$ will lead to smaller value of error rates, but it is not found to be same. This interesting fact about the threshold value of fading parameter $m$ has also been reported for frequency selective fading Nakagami-m channel Zheng et al. [9].

We have further analyzed OFDM system using QPSK as shown in Figure 3. Results obtained from simulations for OFDM-QPSK systems are similar in nature to that of OFDM-BPSK system. BER starts decreasing with increasing value of $\mathrm{m}$. It is very well depicted from graph the threshold value of $m=1.4$ is achieved with BER value of $10^{-2}$. If value of $\mathrm{m}$ is further increased, BER starts increasing. In both the graphs, the BER at value of $m=1$ reports to Rayleigh fading error rate for OFDM systems, which has already been reported in literature.

Finally, it is concluded that BER performance of OFDM system in Nakagami-m channel degrades if we increase $m$ beyond the certain threshold value [18]. 


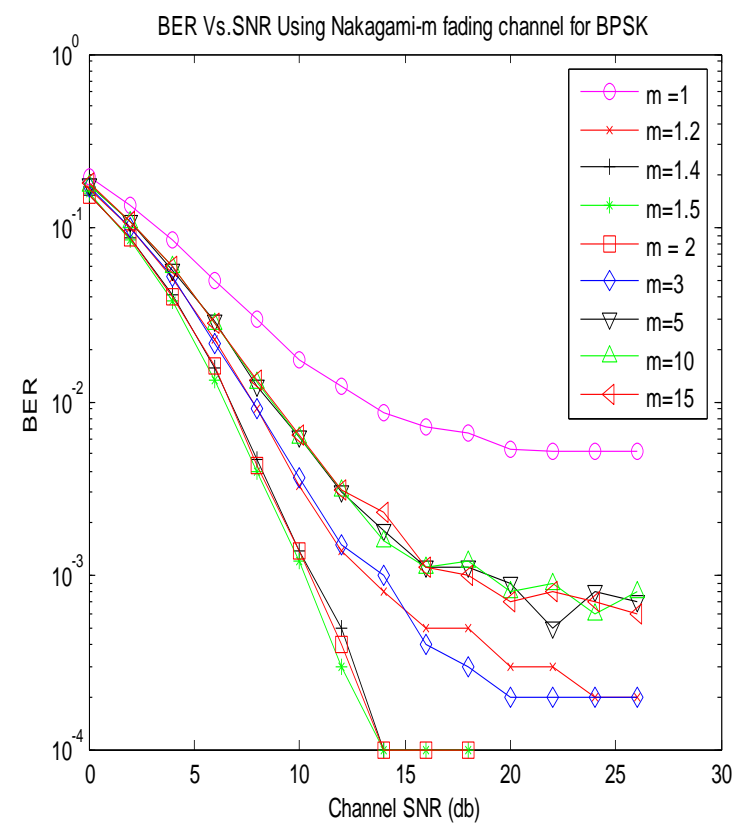

Figure 2. BER vs. SNR for OFDM-BPSK System

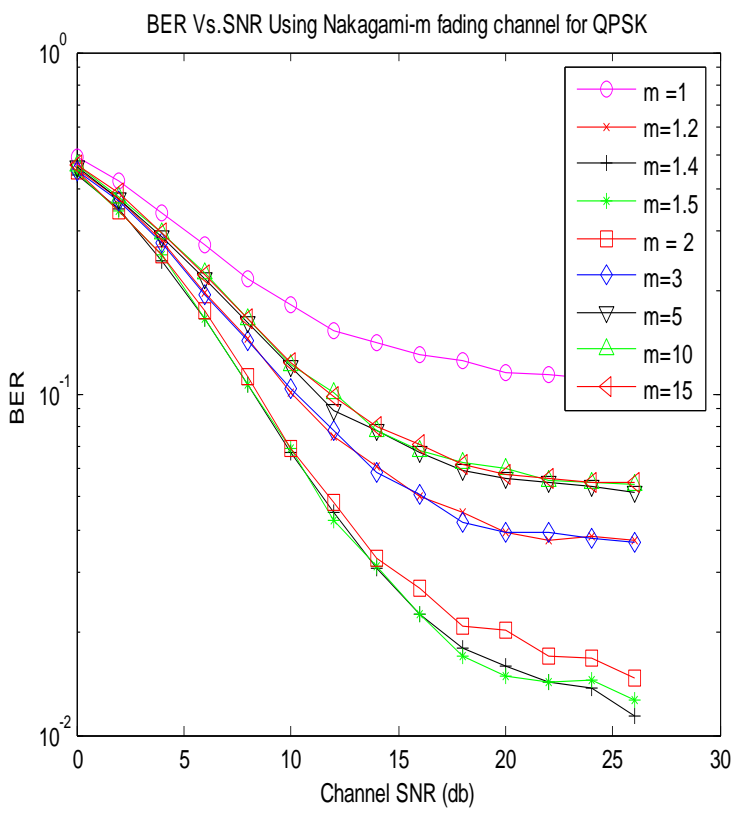

Figure 3. BER vs. SNR for OFDM-QPSK System

\section{Case II: Under the influence of Generalized Gamma fade}

As per discussion in section 2, it is clear that generalized Gamma fade is more flexible since it contains two fading parameters $v$ and $m$. So, in order to evaluate the performance of OFDM system in Gamma fading, we must use the optim value of fading parameterm $=1.4$. Since, it is reported in literature that envelope of Generalized Gamma fading can be obtained through Equation 8 for only discrete values of $\mathrm{m}$ [13], so, we have chosen the discrete value of $m=2$ to evaluate the performance of OFDM system.

Figure 3 and Figure 4 clearly shows that at $v=1$, Generalized Gamma fading distribution behaves like Nakagami-m distribution as stated in literature. As we increases, the value of fading parameter $v$, non-linearity started playing its role and BER is further decreased. However, results obtained with QPSK are more significant compared to BPSK. Improvement in lower SNR is clearly indicated in graphs.

For QPSK system,in Figure 4, the BER at SNR of $15 \mathrm{db}$ for Nakagami-m channel has been reported to close to $10^{-2}$, whereas, in Generelaized Gamma fading channel, BER is reported to be around $10^{-4}$. It clearly shows the severity of fading present in Nakagami-m distribution and flexibilty of Generalized Gamma fading channel.

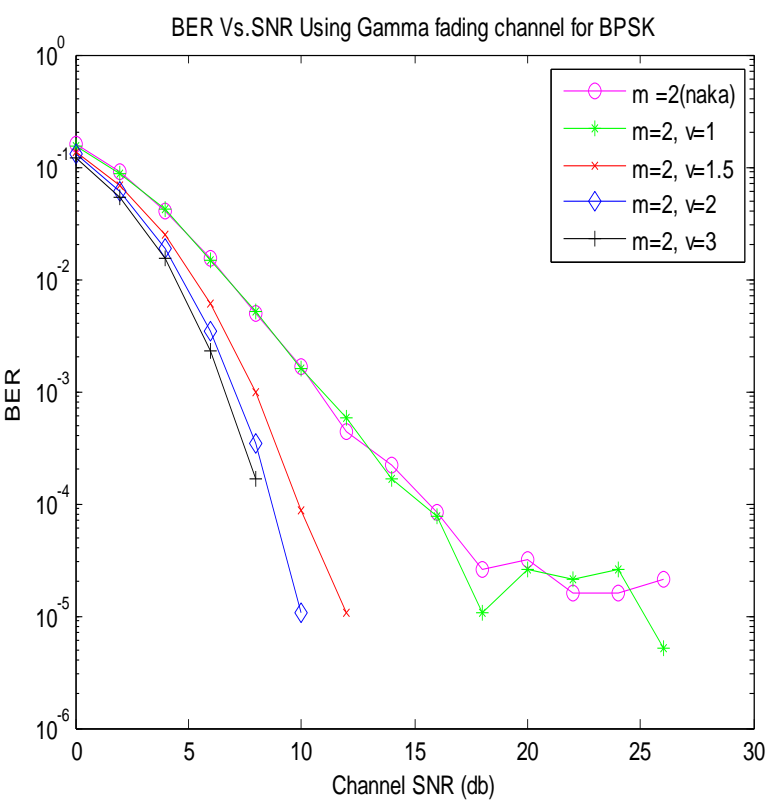

Figure 3. BER vs. SNR for OFDM-BPSK System

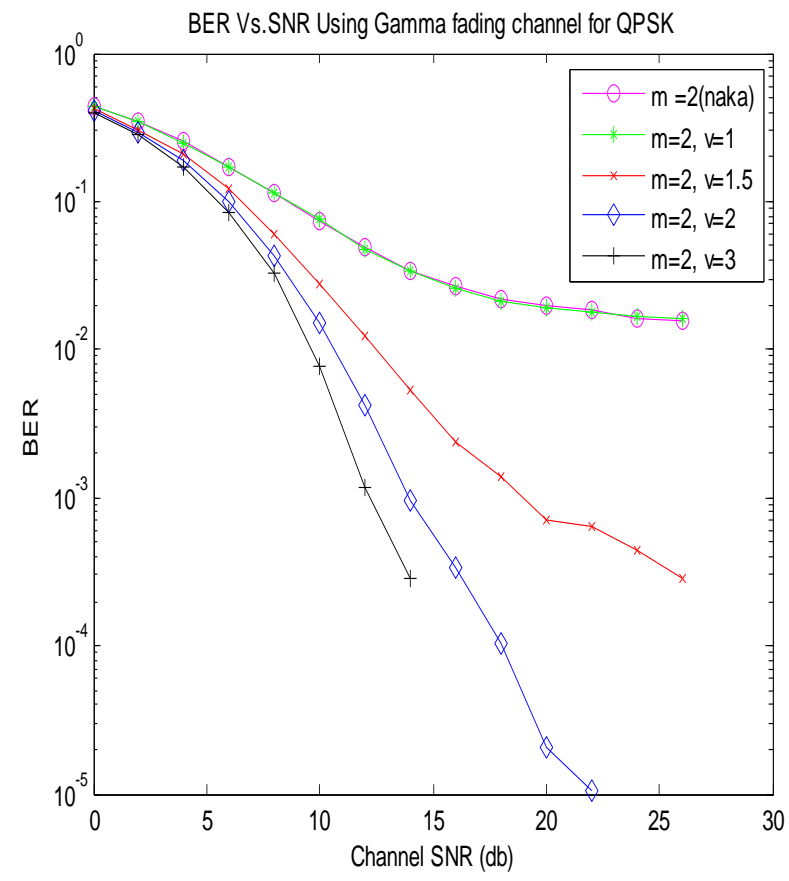

Figure 4. BER vs. SNR for OFDM-BPSK System 


\section{Conclusions}

In this paper, we have evaluated the performance of OFDM system using BPSK and QPSK with OFDM using Generalized Gamma fading distribution. Graphical results show the improvement in OFDM-BPSK and-QPSK system compared to its performance in Nakagami-m fading channel. So, here the versatility of Generalized Gamma model of using the two fading parameters has been proved. The reported BER can be further reduced by using channel estimation or suitable diversity scheme.

\section{REFERENCES}

[1]. Lei Wan, V. K. Dubey, "Bit error probability of OFDM system over frequency nonselective fast Rayleigh fading channels," IEEE Electronics letters, Vo. 36, No. 15, pp13061307, July 2000.

[2]. Jun Lu, Thiang Tjhung,Fumiyuki Adachi and Cheng Li Huang, "BER performance of OFDM-MDPSK system in Frequency -Selective Rician Fading with Diversity Reception," IEEE Trans. On Vehicular Technology, vol. 49, no. 4, pp. 1216-1225, July 2000.

[3]. Yong Jae Ryu, Dong seong Han, "Timing phase estimator overcoming rayleigh fading for OFDM systems," IEEE Proc., pp. 66-67, 2001.

[4]. Sinem Coleri, Mustafa Ergen,Anuj Puri, Ahmad Bahai, "A Study of Channel Estimation in OFDM Systems," IEEE VTC, Vancouver, Canada, vol. 2,pp 894-898, September, 2002.

[5]. Zhiqiang Liu, Georgios B. Giannaki , "Block Differentially Encoded OFDM With Maximum Multipath Diversity," IEEE Transactions on wireless communications, Vol.. 2, No. 3, pp. 420-423, May 2003.

[6]. M. Nakagami, "The m-distribution-A general formula of intensity distribution of rapid fading," in Statistical Methods in Radio Wave Propagation, W. C. Hoffman, Ed. Elmsford, NY: Pergamon, 1960

[7]. Zhein gjiu Kang, Kung Yao, Flavio Lorenzelli, "Nakagami-m Fading Modeling in the Frequency Domain for OFDM system analysis," IEEE Communication letters, vol. 7, no.10, pp. 484-486, Oct.2003.

[8]. Zheng du,Julian Cheng and Norman c. Beaulieu, "Asymptotic BER performance of OFDM in Frequency Selective Nakagami-m Channels," IEEE Conference on Vehicular Technology, vol. 1, pp. 612-615,Sept. 2004.
[9]. Zheng du,Julian Cheng and Norman c. Beaulieu, "Accurate Error Rate Performance Analysis of OFDM on Frequency Seelective Nakagami-m Fadiing Channels," IEEE Trans.on communications.vol. 54, no. 2, pp. 319-328, Feb. 2006.

[10].Zhangyong Ma and Young-il- Kim, "A Novel OFDM receiver in Flat Fading Channel," IEEE Conference on advanced communication technology, ICACT, Vol.. 2, pp. 1052-54, 2005.

[11].SONG Lijun, TANG Youxi, LI Shaogian and HUANG Shunji, "BER Performance of Frequency Domain Differential Demodulation OFDM in Flat Fading Channel," Journal of Electronic Science and Technology of China, Vol. 1, no. 1, Dec. 2003.

[12].Basel Rihawi and Yves Louet, "Gaussian and Flat Rayleigh Fading Channel Influences on PAPR Distribution in MIMOOFDM Systems," 13th European Wireless Conference, Paris,April 2007.

[13]. Aalo VA, Piboongungon T and C D Iskander , 'Bit-error rate of binary digital modulation schemes in generalized gamma fading channels," IEEE Communication Letters, Vol. 09, No. 2, pp 139-141, Feb. 2005

[14].Ferkan yilmaz and Oguz Kucur, "Performance of QS-CDMA over Frequency Selective Time Non-Selective Multipath Generalized Gamma Fading Channels," 13th European Wireless Conference, Paris,April 2007.

[15].Jyoteesh Malhotra, Ajay K Sharma and R S Kaler, "On the Performance Analysis of Wireless Receiver using Generalized-Gamma Fading Model, in annals of telecommunications-annales des télécommunications, international journal, Springer, Vol. 64, No 1-2, pp. 147-153, January-February 2009.

[16]. Ruhallah Ali Hemmati, Paeiz Azmi, "Clipping distortion mitigation in OFDM systems over fading channels by using DFT-based method" Elsevier Computers and Electrical Engineering, vol.31, issue 7, pp 431-443, Oct. 2005.

[17].A.J. Coulson, A.G. Williamson, and R.G. Vaughan, “ Improved Fading Distribution for Mobile Radio," IEE Proc. F-Communication, vol. 145, pp. 197-202, June 1998.

Neetu Sood, Ajay K Sharma, Moin Uddin, "BER Performance of OFDM-BPSK and -QPSK over Nakagami-m Fading Channels," Proc. Of 2nd IEEE International Advance Computing Conference, IACC-2010, pp 88-90, Feb. 2010. 